\title{
Clinical application of a rapid lung-orientated immunoassay in individuals with possible tuberculosis
}

\author{
R A M Breen, ${ }^{1,2}$ S M Barry, ${ }^{1,2}$ C J Smith, ${ }^{3}$ R J Shorten, ${ }^{4}$ J P Dilworth, ${ }^{2}$ I Cropley, ${ }^{2}$ \\ T D McHugh, ${ }^{4}$ S H Gillespie, ${ }^{4}$ G Janossy, ${ }^{1}$ M C I Lipman ${ }^{2}$
}

\begin{abstract}
${ }^{1}$ Department of Immunology, Royal Free and University College Medical School, London, UK; ${ }^{2}$ Departments of Thoracic and HIV Medicine, Royal Free Hospital, London, UK;

${ }^{3}$ Department of Primary Care and Population Science, Royal Free and University College Medical School, London, UK; ${ }^{4}$ Centre for Medical Microbiology, Royal Free and University College Medical School, London, UK
\end{abstract}

Correspondence to: Dr R A M Breen, Department of Thoracic and HIV Medicine, Royal Free Hospital, London NW3 20G, UK; r.breen@ doctors.org.uk

Received 25 June 2007 Accepted 27 June 2007 Published Online First 2 August 2007

\section{ABSTRACT}

Background: Immunological ex vivo assays to diagnose tuberculosis (TB) have great potential but have largely been blood-based and poorly evaluated in active TB. Lung sampling enables combined microbiological and immunological testing and uses higher frequency antigenspecific responses than in blood.

Methods: A prospective evaluation was undertaken of a flow cytometric assay measuring the percentage of interferon- $\gamma$ synthetic CD4+ lymphocytes following stimulation with purified protein derivative of Mycobacterium tuberculosis (PPD) in bronchoalveolar lavage fluid from 250 sputum smear-negative individuals with possible TB. A positive assay was defined as $>1.5 \%$.

Results: Of those who underwent lavage and were diagnosed with active TB, 95\% (106/111) had a positive immunoassay (95\% Cl 89\% to 98\%). In 139 individuals deemed not to have active TB, 105 (76\%) were immunoassay negative (95\% $\mathrm{Cl} 68 \%$ to $82 \%)$. Of the remaining $24 \%$ (34 cases) with a positive immunoassay, a substantial proportion had evidence of untreated TB; in two of these active TB was subsequently diagnosed. Assay performance was unaffected by HIV status, disease site or BCG vaccination. In culture-positive pulmonary cases, response to PPD was more sensitive than nucleic acid amplification testing (94\% vs $73 \%$ ). The use of early secretory antigen target-6 (ESAT-6) responses in 71 subjects was no better than PPD, and 19\% of those with culture-confirmed TB and a positive PPD immunoassay had no detectable response to ESAT-6.

Conclusions: These findings suggest that lung-orientated immunological investigation is a potentially powerful tool in diagnosing individuals with sputum smear-negative active TB, regardless of HIV serostatus.

Koch first used immune reactivity to aid tuberculosis (TB) diagnosis in the enduring form of the tuberculin skin test (TST). ${ }^{1}$ Assays measuring interferon- $\gamma$ (IFN $\gamma$ ) production from blood in response to $\mathrm{TB}$ antigens have recently begun to supersede TST in diagnosing latent $\mathrm{TB}^{2{ }^{3}}$ Their value in advanced HIV and in unselected cases with active TB remains to be established. ${ }^{4}$ Furthermore, a blood-based approach cannot be combined with current microbiological techniques which require samples from the site of disease.

TB is spread primarily by inhalation of organisms into the lung, and this is reflected immunologically by the lung-compartmentalised TB antigen-specific responses that have been observed in TST-positive healthy household contacts of TB and individuals with active TB. ${ }^{5-7}$ Small clinical studies using bronchoalveolar lavage (BAL) have suggested that these phenomena may be used in a clinical setting to make a rapid diagnosis in cases of extrapulmonary and HIV-related TB with greater sensitivity than if blood was used. ${ }^{8-10}$ We therefore sought to determine the diagnostic utility of our approach in all patients at our centre in whom TB was felt to be a possible diagnosis and who were investigated by means of clinically indicated BAL.

\section{METHODS}

\section{Patients and samples}

The study was approved by the Royal Free Hospital ethics committee. All sputum smear-negative adults in whom TB was felt to be a possible diagnosis and who were undergoing a clinically indicated BAL were eligible for inclusion. No subject had commenced antituberculosis medication at the time of BAL Subjects were recruited from outpatient clinics and inpatient wards of our urban teaching hospital between January 2001 and December 2005.

Historically, Bacillus Calmette Guerin (BCG) vaccination was routinely administered in the UK and the recorded rate among the combined native and foreign-born adult population seen in our service remains consistently around $80 \%$.

BAL was performed on the basis of clinical indication as determined by the physician responsible for patient care. A standard technique was used in which a flexible bronchoscope was wedged into a subsegmental bronchus. Aliquots of normal saline to a total volume of $180 \mathrm{ml}$ were introduced into an area of lung showing possible disease on radiological examination or the right middle lobe.

\section{Immunological investigation}

BAL fluid was processed within $2 \mathrm{~h}$ and aliquots sent for routine investigation; $10-20 \mathrm{ml}$ were retained for this study. Absolute CD45+ leucocyte and lymphocyte numbers and the percentages of CD3+, CD4+ and CD8+ T cells were assessed by flow cytometry. ${ }^{11}$ Detection of antigen-specific IFN $\gamma$ synthesis following incubation for $16 \mathrm{~h}$ (overnight) was performed as previously described. ${ }^{8}$ In brief, $5 \times 10^{4}$ CD4 lymphocytes were incubated with tissue culture medium (TCM) alone (negative control); TCM containing $10 \mu \mathrm{g}$ purified protein derivative of Mycobacterium tuberculosis (PPD) (Mycos Research, Colorado, USA); or TCM containing $10 \mu \mathrm{g}$ recombinant early secretory antigen target-6 (ESAT-6) (Colorado State 

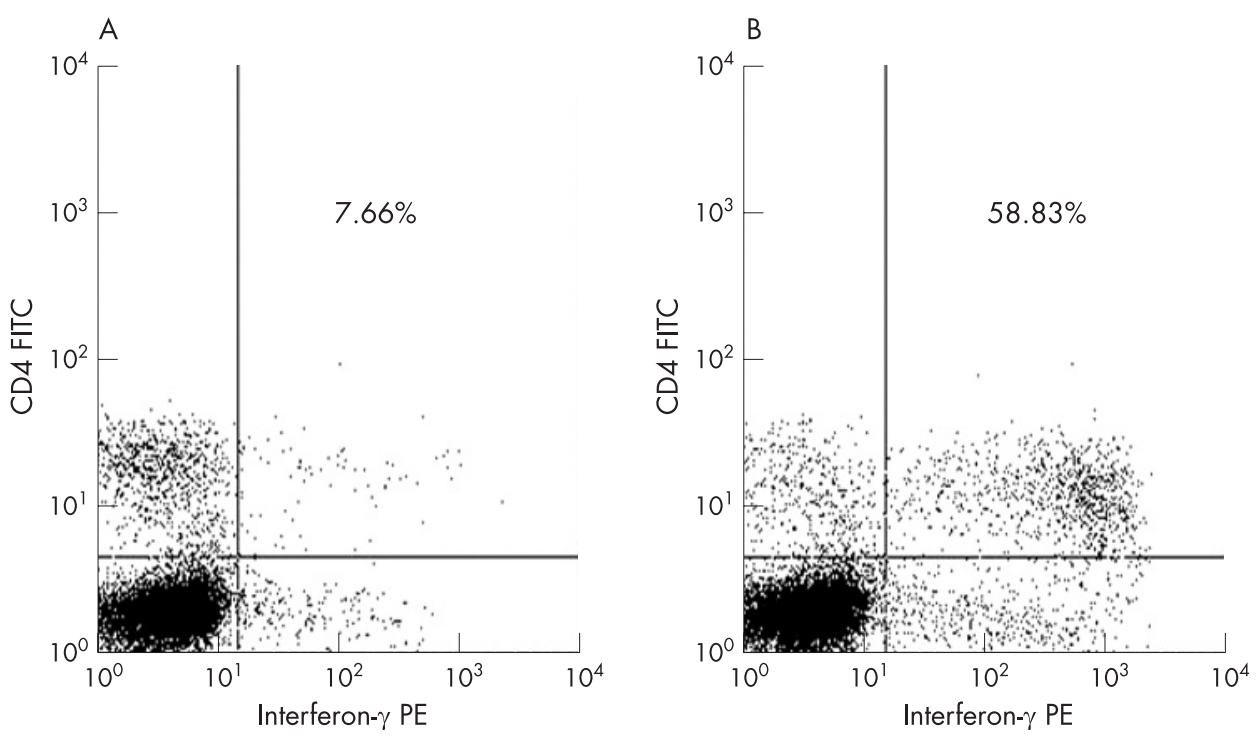

Figure 1 Cytometric dot plots from an HIV-infected individual with a peripheral blood CD4 count of 51 cells/ $\mu$ and active tuberculosis, demonstrating interferon (IFN) $\gamma$ synthesis in response to overnight incubation of induced sputum with purified protein derivative of Mycobacterium tuberculosis (PPD). (A) Proportion of CD4+ lymphocytes producing IFN $\gamma$ after incubation with no antigen added. (B) Proportion of CD4+ lymphocytes producing IFN $\gamma$ after incubation with PPD. The value observed in the negative control was subtracted from that in the PPD-stimulated culture to give a percentage value of PPD-specific CD4+ IFN $\gamma+$ lymphocytes (\%PPD+CD4+IFN $\gamma+$ ) of $51.17 \%$.

University). Brefeldin A (Sigma) was added after 2 h. Harvested cells were fixed and permeabilised (Fix-and-Perm; An-der-Grub) and then stained for expression of $\mathrm{CD} 3, \mathrm{CD} 4$ and IFN $\gamma$ at $4^{\circ} \mathrm{C}$ for 20 min. Stained lymphocytes, identified by flow cytometry scatter, were gated according to CD3 expression to produce a histogram of IFN $\gamma$ producing CD4+ and $\mathrm{CD} 4-\mathrm{T}$ cells. The frequency of IFN $\gamma+\mathrm{CD} 4+$ lymphocytes as a percentage of the total CD4+ T lymphocyte population (\%CD4+IFN $\gamma+$ ) was determined (fig 1). The value observed in the negative control was subtracted from that in the PPD-stimulated culture to give a percentage value of PPD-specific CD4+ IFN $\gamma+$ lymphocytes (\%PPD+CD4+IFN $\gamma+$ ).

\section{Microbiological diagnosis}

Following decontamination and concentration by centrifugation at $3000 \mathrm{~g}$ for $30 \mathrm{~min}$, BAL specimens were divided. Aliquots were prepared for microscopy and direct nucleic acid amplification testing (NAAT) using the $\mathrm{BD}$ Probetec ctb assay (TBSDA). ${ }^{12}$ The remainder was washed, centrifuged again and then inoculated into the MB BacT ALERT liquid culture system (bioMérieux UK, Hampshire, UK). Microscopy smears were stained with auramine- $O$ and the positive smears were confirmed using a Ziehl-Neelsen stain. The isolate was confirmed as $M$ tuberculosis by the regional Mycobacterial Reference Laboratory.

\section{Diagnostic definition}

A diagnosis of TB was accepted if (1) $M$ tuberculosis was cultured or (2) if a clinical diagnosis was made, there was good evidence for this based on radiology or histology with an appropriate response to treatment. Diagnostic decisions were made without reference to immunological data.

\section{Statistical assessment}

Comparisons between groups were made using the Wilcoxon test; $95 \%$ confidence intervals (CI) were calculated using the exact Wilson method. The data were analysed using SAS Version 8.2 (SAS Institute Inc, Cary, NC, USA).

\section{RESULTS}

\section{Patient information}

Two hundred and fifty individuals of median age 40 years (range 19-64) underwent BAL; 41\% were Black, 35\% Caucasian, $21 \%$ Asian and 3\% others. Sixty-three (25\%) were HIV-infected with a median blood CD4 count of 153 cells/ $\mu \mathrm{l}$ (range 5-1065).

In total, 111 of the 250 individuals $(44 \%)$ had a diagnosis of active TB of whom $21 \%(n=23)$ were co-infected with HIV (median blood CD4 count 153 cells/ $\mu$ l (range 10-1065)). Eightythree of the $111(75 \%)$ were culture positive (73 from the same sample as used for immunology; 10 from a sample other than BAL fluid) and $28(25 \%)$ received a clinical diagnosis of active TB. Of the $139(56 \%)$ in whom active TB was not diagnosed, the commonest alternative definitive diagnosis in those not infected with HIV was sarcoidosis (22/100), and in those infected with HIV Pneumocystis jirovecii pneumonia (12/39) was the most common alternative diagnosis.

In the total group, the median percentage of CD45+ BAL leucocytes that were lymphocytes was $23.2 \%$ (range 1.2-85.2). Among the CD3+ lymphocytes, the ratio of $\mathrm{CD} 4+$ to $\mathrm{CD} 8+$ lymphocytes (CD4:CD8) was 1.25 (range 0.01-44.10).

\section{PPD-specific IFN $\gamma$ responses in the lung compared with the final diagnosis}

In the $\mathrm{TB}$ group the median percentage of PPD-specific CD4+IFN $\gamma$ synthetic lymphocytes (\%PPD+CD4+IFN $\gamma+$ ) was $14.63 \%$ (range 0-79.11) for those with culture-proven TB and $10.41 \%$ (range $0.19-70.50$ ) for those with clinical diagnoses $(p=0.36)$ compared with $0.21 \%$ (range $0-27.10$ ) in the non-TB group $(p<0.001)$. HIV infection did not significantly alter these values (HIV/TB: 8.89\% (range 0-61.04); TB alone: $14.63 \%$ (range $0.12-79.11), \mathrm{p}=0.24$ ).

\section{PPD-specific lung immunoassay as a diagnostic tool}

Using a receiver operator characteristic (ROC) curve (area under the curve $=0.908$ ), we assessed that a $\% \mathrm{PPD}+\mathrm{CD} 4+\mathrm{IFN} \gamma+$ value of $1.5 \%$ would provide a simple and appropriate assay 


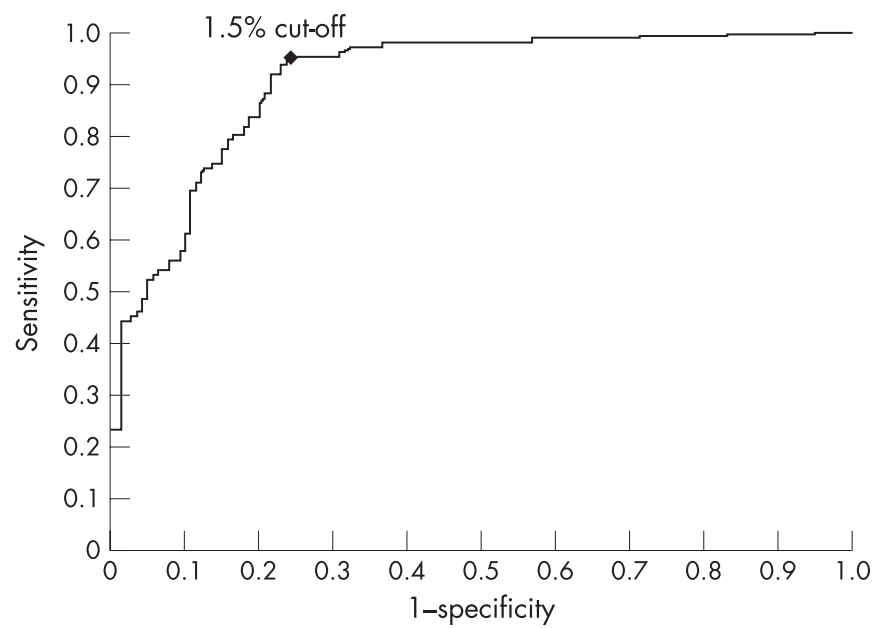

Figure 2 Receiver operator characteristic (ROC) curve for cut-off values of \%PPD+CD4+IFN $\gamma+$ assay results versus final diagnosis of tuberculosis.

cut-off value (fig 2). The sensitivity of a \%PPD+CD4+IFN $\gamma+$ value of $\geqslant 1.5 \%$ for a diagnosis of active TB was $95 \%(95 \%$ CI $89 \%$ to $98 \%$ ) and the specificity was $76 \%$ (95\% CI $68 \%$ to $82 \%$; table 1).

A $\%$ PPD + CD $4+$ IFN $\gamma+$ value of $\geqslant 1.5 \%$ was observed in $95 \%$ (79/83) with culture-positive TB and 93\% (26/28) who received a clinical diagnosis of active TB. These values were 95\% (90/95) in subjects with pulmonary TB and $100 \%(16 / 16)$ in whom the active disease site was felt in the end to be non-pulmonary.

\section{PPD-specific immunoassay versus nucleic acid amplification test (NAAT) in BAL}

The immunoassay was compared with a NAAT in 67 cases in which $M$ tuberculosis was grown from BAL fluid. This ensured that TB DNA was present for amplification. Both the TB-SDA and the immune-based test were highly sensitive in smearpositive BAL fluid (table 2). However, unlike the TB-SDA, the performance of the immune test was maintained when the smear was negative. The results of this comparison and further analysis according to whether the BAL fluid was acid and alcohol fast bacilli (AAFB) smear negative or positive are also shown in table 2 .

\section{PPD-specific IFN $\gamma$ responses in the lung discordant with final diagnosis}

Six of 111 individuals (5\%) who received a final diagnosis of TB had an observed \%PPD+CD4+IFN $\gamma+$ value of $<1.5 \%$. No consistent pattern of disease was observed in this group; and one had a \%PPD+CD4+IFN $\gamma+$ value of $12.25 \%$ observed in pleural fluid.
Thirty-four of 139 individuals (24\%) not diagnosed with TB had a positive immunoassay. Of these 11 were of Asian ethnicity (all born outside the UK), 11 were Black (all born outside the UK), 12 were Caucasian ( 8 with risk factors for TB due to either drug or alcohol use, occupation, country of birth or known contact with an index case). Four of 34 had mycobacteria other than tuberculosis (MOTT) cultured from BAL fluid (1 grew $M$ fortuitum but with a positive NAAT for $M$ tuberculosis; 1 grew $M$ kansasii; 2 grew $M$ avium complex, 1 with a positive NAAT for $M$ tuberculosis). Only 1 individual had definitely received a previous course of antituberculosis therapy. During a median follow-up period of 33 months (range 12-67), 2/34 subjects (6\%) developed active TB (diagnosed 12 and 23 months after initial BAL, respectively). These subjects are further described in table 3 .

\section{ESAT-6-specific IFN $\gamma$ responses in the lung versus final diagnosis}

Seventy-one consecutive individuals from the BAL study population had samples stimulated with recombinant ESAT-6 in addition to PPD. Of these, 32 (45\%) were diagnosed with active TB (27 culture proven and 5 clinical). The median (range) percentage of ESAT-6-specific CD4+IFN $\gamma$ synthetic lymphocytes in these 32 subjects was $1.02 \%(0-12.39)$. No significant difference was seen between individuals with culture proven or clinically diagnosed $\mathrm{TB}$ and those who had a positive PPD immunoassay but were not diagnosed with TB (1.28\% (0-12.39) vs $0.78 \%(0.19-6.24)$ vs $0.72 \%(0-3.88)$, respectively). In the TB culture positive group, $5 / 27$ (19\%) had no measurable response to ESAT-6 despite all 5 having a positive PPD immunoassay.

\section{DISCUSSION}

These results demonstrate the potential of a lung-orientated immunological approach to the diagnosis of active TB using BAL and flow cytometry in a large unselected series. Unlike current blood-based immunoassays, BAL may also provide microbiological confirmation of the rapid immunological test results. In 250 sputum smear-negative individuals in whom TB was considered to be a possible diagnosis, the PPD-based IFN $\gamma$ immune assay had a sensitivity of $95 \%$ and specificity of $76 \%$, with comparable utility in subjects with and without HIV infection and in those with pulmonary and non-pulmonary TB.

Our immunological approach had markedly superior overall sensitivity compared with a commercially available NAAT representative of current rapid microbiological diagnostics. In particular, our immunoassay showed no diminution in performance with BAL smear-negative culture-positive cases, suggesting significant advantages for immunological assays when there is a lower mycobacterial burden or minimal pulmonary disease. This agrees with the limited data available on the value of molecular techniques in such cases. ${ }^{13}{ }^{14}$

Table 1 Application of a bronchoalveolar lavage (BAL) immunoassay for the diagnosis of tuberculosis (TB) using interferon- $\gamma$ synthetic responses of CD4+ lymphocytes to purified protein derivative of Mycobacterium tuberculosis (PPD)

\begin{tabular}{lllll}
\hline & $\begin{array}{l}\text { TB culture } \\
\text { positive } \\
(\mathbf{n}=\mathbf{8 3})\end{array}$ & $\begin{array}{l}\text { Clinical diagnoses } \\
\text { of TB } \\
(\mathbf{n}=\mathbf{2 8})\end{array}$ & $\begin{array}{l}\text { Final diagnosis } \\
\text { of TB } \\
(\mathbf{n}=\mathbf{1 1 1})\end{array}$ & $\begin{array}{l}\text { Final diagnosis } \\
\mathbf{n o t} \text { TB } \\
(\mathbf{n}=\mathbf{1 3 9})\end{array}$ \\
\hline $\begin{array}{l}\text { Positive BAL immunoassay } \\
(\mathbf{n}=\mathbf{1 3 9})\end{array}$ & $95 \%(79)$ & $93 \%(26)$ & $95 \%(105)$ & $24 \%(34)$ \\
$\begin{array}{l}\text { Negative BAL immunoassay } \\
(\mathbf{n}=\mathbf{1 1 1})\end{array}$ & $5 \%(4)$ & $7 \%(2)$ & $5 \%(6)$ & $76 \%(105)$ \\
\hline
\end{tabular}


Table 2 Comparison of bronchoalveolar lavage (BAL) smear for acid and alcohol fast bacilli (AAFB), nucleic acid amplification testing (TB-SDA) and a PPD-based immunoassay as diagnostic tools in Mycobacterium tuberculosis culture positive individuals

\begin{tabular}{llll}
\hline & $\begin{array}{l}\text { BAL AAFB } \\
\text { smear positive }\end{array}$ & TB-SDA positive & $\begin{array}{l}\text { Positive BAL } \\
\text { immunoassay }\end{array}$ \\
\hline BAL AAFB smear positive & $100 \%(30 / 30)$ & $97 \%(29 / 30)$ & $93 \%(28 / 30)$ \\
BAL AAFB smear negative & $0 \%(0 / 37)$ & $54 \%(20 / 37)$ & $95 \%(35 / 37)$ \\
Total & $45 \%(30 / 67)$ & $73 \%(49 / 67)$ & $94 \%(63 / 67)$ \\
\hline
\end{tabular}

A significant drawback of the tuberculin skin test is that positive responses are often generated by Bacille-CalmetteGuerin (BCG) vaccinated individuals with no exposure to $M$ tuberculosis. ${ }^{15}$ This has prompted the use of antigens encoded in the region of difference 1 (RD1) portion of the genome of $M$ tuberculosis such as ESAT-6 which are absent in BCG strains. ${ }^{16}{ }^{17}$ We found that ESAT- 6 provided no further information than
PPD lymphocyte stimulation. Furthermore, in our largely BCGvaccinated population, responses to PPD in the lung due to BCG were not detectable. The most plausible explanation is that BCG-specific memory lymphocytes traffic through blood and home to the skin while avoiding the lung. ${ }^{18}$

We did observe a significant number of positive tests in subjects not clinically felt to have active TB. We believe that a

Table 3 Individuals with a positive immunoassay but not diagnosed with active tuberculosis following bronchoalveolar lavage (BAL) and associated investigations (those diagnosed with active disease during follow-up are marked in bold type)

\begin{tabular}{|c|c|c|}
\hline $\begin{array}{l}\text { BAL \%PPD+ } \\
\text { CD4+IFN } \gamma+\end{array}$ & Ethnicity & Comments \\
\hline $1.64 \%$ & Caucasian & Right pleural effusion of uncertain aetiology; HIV-infected \\
\hline $1.82 \%$ & Asian & Mediastinal lymphadenopathy; clinical diagnosis of sarcoidosis \\
\hline $2.02 \%$ & Black-African & LUL mass of uncertain aetiology \\
\hline $2.07 \%$ & Asian & $\begin{array}{l}\text { BAL TB NAAT positive; } M \text { fortuitum grown; received anti-TB therapy for } 2 \text { months and } \\
\text { then discontinued }\end{array}$ \\
\hline $2.34 \%$ & Black-African & Clinical diagnosis of pulmonary sarcoidosis \\
\hline $2.63 \%$ & Caucasian & Haemoptyis of uncertain aetiology \\
\hline $2.98 \%$ & Asian & RML collapse and multiple pulmonary nodules \\
\hline $2.99 \%$ & Asian & Pulmonary and cutaneous nodules; clinical diagnosis of sarcoidosis \\
\hline $3.23 \%$ & Caucasian & Multiple pulmonary nodules of uncertain aetiology; chronic alcohol use \\
\hline $3.44 \%$ & Caucasian & Calcified mediastinal lymph nodes of uncertain aetiology \\
\hline $3.47 \%$ & Black-African & $\begin{array}{l}\text { Mycobacterium avium complex cultured from BAL BAL TB NAAT positive; HIV- } \\
\text { infected }\end{array}$ \\
\hline $3.55 \%$ & Black-African & Haemoptysis; household contact of smear positive TB \\
\hline $3.88 \%$ & Caucasian & Injecting drug user with pulmonary nodules of uncertain aetiology; HIV-infected \\
\hline $4.75 \%$ & Asian & Clinical diagnosis of pulmonary sarcoidosis \\
\hline $4.78 \%$ & Caucasian & $\begin{array}{l}\text { Right hilar and mediastinal lymphadenopathy with granulomas on biopsy; clinical } \\
\text { diagnosis of sarcoidosis }\end{array}$ \\
\hline $5.03 \%$ & Black-African & LUL nodules of uncertain aetiology; HIV-infected \\
\hline $5.64 \%$ & Black-African & $\begin{array}{l}\text { RML consolidation and calcified mediastinal lymph nodes; TST +ve (Heaf grade 4); } \\
\text { chronic cough of unknown cause }\end{array}$ \\
\hline $6.07 \%$ & Asian & Left hilar lymphadenopathy; no diagnosis made \\
\hline $6.61 \%$ & Caucasian & Right pleural effusion of uncertain aetiology; HCW with occupational TB exposure \\
\hline $8.52 \%$ & Caucasian & Calcified pulmonary nodules and apical scarring on CXR \\
\hline $10.79 \%$ & Caucasian & TST +ve (Heaf grade 4); apical and pleural shadowing on CXR \\
\hline $11.48 \%$ & Caucasian & Disseminated $M$ kansasii with common variable immunodeficiency \\
\hline $12.00 \%$ & Black-African & Clinical diagnosis of pulmonary sarcoidosis \\
\hline $12.78 \%$ & Black-African & Pulmonary nodules of uncertain aetiology \\
\hline $13.00 \%$ & Caucasian & Mediastinal lymphadenopathy of uncertain cause; Kosovan \\
\hline $13.24 \%$ & Black-African & $\begin{array}{l}\text { Pulmonary nodules and axillary, abdominal and mediastinal lymph nodes; HIV- } \\
\text { infected; no diagnosis made }\end{array}$ \\
\hline $13.90 \%$ & Caucasian & Bilateral hilar lymphadenopathy; clinical diagnosis of sarcoidosis \\
\hline $14.49 \%$ & Black-African & Pericardial thickening; HCW with occupational TB exposure \\
\hline $15.20 \%$ & Black-African & Clinical diagnosis of sarcoidosis with good response to corticosteroids \\
\hline $15.64 \%$ & Caucasian & TST +ve (Heaf grade 4). HCW with occupational exposure to TB \\
\hline $16.12 \%$ & Asian & $\begin{array}{l}\text { Mycobacterium avium complex cultured from BAL fluid; probable immune } \\
\text { reconstitution illness on starting antiretroviral agents; HIV-infected }\end{array}$ \\
\hline $16.32 \%$ & Asian & Mediastinal lymphadenopathy of uncertain cause \\
\hline $26.74 \%$ & Asian & RML consolidation and calcified mediastinal lymph nodes \\
\hline $27.10 \%$ & Asian & $\begin{array}{l}\text { Previous anti-TB treatment for pulmonary disease; no clinical evidence of current } \\
\text { activity }\end{array}$ \\
\hline
\end{tabular}

BAL, bronchoalveolar lavage; CXR, chest radiograph; HCW, health care worker; LUL, left upper lobe; NAAT, nucleic acid amplification technique; RML, right middle lobe; TST, tuberculin skin test. 
substantial proportion of these subjects in fact had good evidence for TB infection (as detailed in table 3), if not current active disease. Given the similar finding with both PPD and ESAT-6, it is probable that all of the current mycobacterial antigens used in TB diagnostics will exhibit these characteristics. ${ }^{10}$ Do these responses therefore have clinical value? It is likely that they are genuinely directed against mycobacteria, some of which are perhaps environmental. However, they may also signify the presence of undetected TB infection with viable organisms, and therefore a risk of later reactivation. ${ }^{19}$ It is noteworthy that, during follow-up of the 34 individuals with such positive results, 2 have developed active TB. The reported poor specificity of immune-based methods must therefore be judged against the inadequacies of both clinical evaluation and other diagnostic techniques. ${ }^{20}$

An important feature of our approach is that the assay had comparable performance in patients with and without HIV coinfection, as well as those with non-pulmonary or pulmonary disease. Lymphocyte recirculation to the original site of $\mathrm{TB}$ infection (usually the lung), regardless of subsequent disease manifestation, may explain why we can detect non-pulmonary TB using lung-based assays. Given the difficulties that exist in diagnosing $\mathrm{TB}$ outside the lung, we feel that this test with its high sensitivity, if used within a suitable clinical pathway, might reduce the need for multiple investigative procedures by "ruling out" TB at an early stage.

Our data strongly suggest that immunological assays, which can be combined with conventional culture and NAAT in BAL samples, have the potential to improve the diagnosis of patients with suspected TB. Simplification of sample collection and processing would enable this technique to be applied beyond its current research setting.

Acknowledgements: Recombinant ESAT-6 was kindly provided as part of NIH NIAID Contract No. HHSN266200400091C entitled "Tuberculosis Vaccine Testing and Research Materials" which was awarded to Colorado State University.

Funding: None

Competing interests: None

\section{REFERENCES}

1. Huebner RE, Schein MF, Bass JB Jr. The tuberculin skin test. Clin Infect Dis 1993;17:968-75.
2. Chapman AL, Munkata M, Wilkinson KA, et al. Rapid detection of active and latent tuberculosis infection in HIV-positive individuals by enumeration of Mycobacterium tuberculosis-specific T cells. AIDS 2000;16:2285-93.

3. Brock I, Weldingh K, Lillebaek T, et al. Comparison of tuberculin skin test and new specific blood test in tuberculosis contacts. Am J Respir Crit Care Med 2004;170:659.

4. Dewan PK, Grinsdale J, Kawamura LM. Low sensitivity of a whole-blood interferongamma release assay for detection of active tuberculosis. Clin Infect Dis 2007;44:6973.

5. Rouillon A, Predrizet S, Parrot R. Transmission of tubercle bacilli: the effects of chemotherapy. Tubercle 1976;57:275-99.

6. Schwander SK, Torres M, Carranza CC, et al. Pulmonary mononuclear cell responses to antigens of Mycobacterium tuberculosis in healthy household contacts of patients with active tuberculosis and healthy. J Immunol 2000;165:1479-85.

7. Schwander SK, Torres M, Sada E, et al. Enhanced responses to Mycobacterium tuberculosis antigens by human alveolar lymphocytes during active pulmonary tuberculosis. J Infect Dis 1998;178:1434-45.

8. Barry SM, Lipman MCl, Bannister B, et al. Purified protein derivative-activated type 1 cytokine-producing CD4+ T lymphocytes in the lung: a characteristic feature of active pulmonary and non-pulmonary TB. J Infect Dis 2003;187:243-50.

9. Breen RAM, Janossy G, Cropley I, et al. Detection of mycobacterial antigen responses in lung but not blood in HIV/tuberculosis co-infected subjects. AIDS 2006;20:1330-2.

10. Jafari C, Ernst M, Kalsdorf B, et al. Rapid diagnosis of smear-negative tuberculosis by bronchoalveolar lavage enzyme-linked Immunospot. Am J Respir Crit Care Med 2006;174:1048-54.

11. Barry SM, Janossy G. Optimal gating strategies for determining bronchoalveolar lavage CD4/CD8 lymphocyte ratios by flow cytometry. J Immunol Methods 2004:285:15-23.

12. McHugh TD, Pope CF, Ling CL, et al. Evaluation of BD ProbeTec Strand displacement amplification (SDA) system for non-respiratory and respiratory samples. J Med Microbiol 2004;53:1215-9

13. Al Zahrani $\mathbf{K}, \mathrm{Al}$ Jahdali $\mathrm{H}$, Poirier $\mathrm{L}$, et al. Accuracy and utility of commercially available amplification and serologic tests for the diagnosis of minimal pulmonary tuberculosis. Am J Respir Crit Care Med 2000;162:1323-9.

14. Soini H, Musser JM. Molecular diagnosis of mycobacteria. Clin Chem 2001;47:80914.

15. Pai M, Riley LW, Colford JM Jr. Interferon-gamma assays in the immunodiagnosis of tuberculosis: a systematic review. Lancet Infect Dis 2004:4:761-76.

16. Harboe M, Oettinger T, Wiker HG, et al. Evidence for occurrence of the ESAT-6 protein in Mycobacterium tuberculosis and virulent Mycobacterium bovis and for its absence in Mycobacterium bovis BCG. Infect Immun 1996;64:16-22.

17. Lalvani A, Pathan AA, McShane $\mathrm{H}$, et al. Rapid detection of Mycobacterium tuberculosis infection by enumeration of antigen-specific T cells. Am J Respir Crit Care Med 2001;163:824-8.

18. Mora JR, von Andrian UH. T-cell homing specificity and plasticity: new concepts and future challenges. Trends Immunol 2006;27:235-43.

19. Grzybowski S, Fishaut $\mathrm{H}$, Rowe J, et al. Tuberculosis among patients with various radiologic abnormalities followed by the chest clinic service. Am Rev Respir Dis 1971;104:605-8.

20. Miller LG, Asch SM, Yu El, et al. A population-based survey of tuberculosis symptoms: how atypical are atypical presentations? Clin Infect Dis 2000;30:293-9.

Keep up to date: sign up for our alerting services

Find out automatically when an article is published on a specific topic or by a particular author. We can also alert you when an article is cited or if an eLetter or correction is published. You can also choose to be alerted when a new issue is published online [and when we post articles Online First]. Check out the New Content Alerts and Citation tracker from the Online tools section on the home page. 